\title{
Del método científico al diagnóstico de problemas de ingeniería en México
}

\author{
O.E. Sotelo-Rosas y L.C. Ramírez-Carrera \\ $M$ aestría en Ciencias en Ingeniería Administrativa \\ División de Estudios de Posgrado e Investigación, Instituto Tecnológico de 0 rizaba \\ E-mail: en_canto@hotmail.com y s_c__a_r@hotmail.com
}

(recibido febrero de 2004; aceptado: octubre de 2004)

\begin{abstract}
Resumen
Si se carece de un sistema claro para realizar el diagnóstico de una situación problemática, habrá un entorpecimiento en el proceso de definición de objetivos, clave en la solución de situaciones de decisión. Por consiguiente, pueden darse soluciones equivocadas que no corresponden a las necesidades que deben atenderse en los sistemas empresariales. La detección de un vacío metodológico en este sentido, se dio como fruto de una revisión a cincuenta tesis de maestría elaboradas entre los años 2000-2004, y a la aplicación de cincuenta encuestas a alumnos de posgrado del Instituto Tecnológico de Orizaba. Este estudio de campo, justifica la elaboración de un método claro, confiable y sencillo, que disminuya la ambigüedad y complejidad presentes cuando se busca la solución de problemas de ingeniería. Se empleó el método de investigación cualitativo, cuyo objetivo científico principal es la comprensión de los fenómenos mediante el análisis de las percepciones e interpretaciones de los sujetos que realizan la investigación. Mediante un discernimiento analógico entre el llamado método científico y el inicio del proceso de decisión, y como parte funda mental de este proyecto, se propone un "Proceso integral de diagnóstico para resolver una situación problemática", empleando herramientas de TQM. EI modelo propuesto, fue validado en una empresa de la región de Orizaba, cuyo informe cae fuera del alcance de este artículo.
\end{abstract}

Descriptores: Método científico, situación problemática, diagnóstico, solución de problemas de ingeniería, administración total de la calidad (TQM)

\begin{abstract}
If it islackeda clear system to maketheprob lem aticsitu a tion di ag nostic, will bean obstruction in thedefinition ofobjectivesprocess, key in thedeci sion situ ationssolu tion. Therefore, mistaken sdu tions can occur, that do not cor respond to the ne ces si ties that must betaken care of in the en ter prise sys tems. The detection of a methodologic emp ti ness in this senseis the fruit of a revi sion to fifty $M$ as ter de greethe ses elab orated between 2000-2004 years, and to theap plica tion of fifty sur veys to posgrado' sstu dents of theTech nological Institute of 0 rizaba. This field study justi fies theelab o ra tion of a surereli ableand sim plemethod that it di min ishes the am bi gu ity and com plex ity when theen gi neer ing prob lems solu tionslook for. Weused thequal itativein vestiga tion method, whosemain sci en tificobjectiveistheun der stand ing of thephenomena by mean so theanal y sis of theper ceptions and in ter preta tions of thepeo plethat makethein vesti gation. By means ofanal ogy discern ment between thesci en tificmethod and thebegin ning of thedeci sion process, and asfun da mental part of this project, weproposean "In tegral Process of di agnosisto solvea problem aticsitu a tion", using TQ M tools. Thepro posed model was val $i$ dated in a re gion of 0 rizaba Com pany, whose re port is out side to the reach of thisarticle.
\end{abstract}

Keywords: Sci en tificmethod, problematicsitu a tion, di agnostic, en gi neer ing prob lems so lu tions, to tal qual ity man agement (TQM ). 


\section{Introducción}

Tomando como base datos obtenidos en un estudio de campo realizado en la División de Estudios de Posgrado e Investigación del Instituto Tecnológico de Orizaba, por medio de: a) una revisión a cincuenta tesis de las maestrías en ciencias en ingeniería administrativa e Ingeniería Industrial y b) encuestas a cincuenta compañeros alumnos de la Maestría en Ingeniería Administrativa; se pudo concluir, si bien parcialmente, que existen vacíos metodológicos en la fase del diagnóstico para la resolución de problemas tecnológicos, ya que el $30 \%$ de los estudiantes encuestados desconocieron con exactitud los pasos para la realización de éste; detectándose también una desinformación en cuanto al desarrollo, aplicación y conclusión de métodos de diagnosis. Los autores creen que carecer de un sistema claro y preciso para especificar un problema, entorpece la adecuada solución del mismo, hablando en términos de economización de recursos. Se requeriría, por lo tanto, de un método funcional, basado en el pensamiento racional, para realizar un diagnóstico de problemas que requieran de la aplicación de la ingeniería.

\section{Marco de referencia}

Una de las cualidades del ser humano, es su tendencia a tratar de comprender y explicar el mundo que lo rodea y buscar el por qué de los fenómenos que observa. Los procesos mentales que sirven de base para la solución de esta inquietud, se fundamentan en una amplia gama de métodos. El más conocido y empleado de ellos es el método científico, iniciado por Fran cis Bacon (1561- 1626), una de las personalidades más sobresalientes de la historia de la ciencia del mundo oc ci den tal moderno.

"Francis Bacon sembró la semilla del método científico al atacar el método deductivo y señalar la necesidad de sustituirlo por la observación directa de los hechos, a partir de la cual podrían extraerse conclusiones generales." (Van Dalen y Meyer, 1979).

La gran cantidad de información producida de la recolección de hechos al azar, propuesta por el método de Bacon resultaba difícil de manejar, por lo tanto, hombres como Galileo Galilei (15641642), René Des cartes (1596-1650) e Isaac New ton (1712-1727), llegaron a superar la sim ple combinación de los procesos inductivo y deductivo, lo cual originó el moderno método científico. Más tarde, John Dewey (1859-1952), contribuye a enriquecer este método haciendo una recolección metódica de hechos que reemplazaron la simple acumulación arbitraria.

En este trabajo, se ha seleccionado la exposición de las cinco etapas del método, desde la visión de Dewey, según Van Dalen y Meyer (1979). La razón de haber elegido a estos autores, es que se ha empleado su explicación en repetidas ocasiones exitosamente para la enseñanza del método científico, el cual se expone a continuación:

"1. Percepción de una dificultad El individuo encuentra algún obstáculo, experiencia o problema que le ocupa, y entonces

a) Carece de los medios para llegar al fin deseado.

b) Tiene dificultad para determinar el carácter de un objeto, o bien,

c) No puede explicar un acontecimiento inesperado.

2. Identificación y definición de la dificultad El individuo efectúa observaciones, registra hechos que le permiten definir su dificultad con mayor precisión.

3. Soluciones propuestas para el problema: hipótesis A partir del estudio preliminar de los hechos, el individuo formula las conjeturas inteligentes acerca de las posibles soluciones del problema.

Las conjeturas -generalizaciones que ofrece para explicar los hechos que originan la dificultadse denominan hipótesis.

4. Deducción de las consecuencias de las soluciones propuestas

Deductivamente, el individuo llega a la conclusión de que, si cada hipótesis es verdadera, le seguirán ciertas consecuencias. 
5. Verificación de las hipótesis mediante la acción

El individuo pone a prueba cada una de las hipótesis buscando hechos observables que permitan confirmar si las consecuencias que deberán seguir, se producen o no. Mediante este procedimiento puede determinar cuál de las hipótesis concuerda con los hechos observables y, de esa manera, hallar la solución más confiable para su problema." (Van Dalen y Meyer, 1979).

Por otro lado, al enfocar el asunto de la solución de problemas desde las disciplinas administrativas, hemos seleccionado a Easton (1978), quien describe un método que consta de tres etapas fundamentales para iniciar un proceso activo de decisión:

a) la percepción de una necesidad de cambio o reconocimiento del problema;

b) el diagnóstico o entendimiento claro de la situación problemática actual y

c) la definición de objetivos y metas de decisión a través de una jerarquía de objetivos; lo cual genera acciones concretas y acertadas para resolver la problemática en cuestión.

El primer paso requiere que un sujeto, a quien se le llama decisor, perciba un mal funcionamiento y/o la obsolescencia de un cierto sistema. La diferencia entre estos dos términos, es que los problemas con mal funcionamiento son factibles de corregir mediante ciertas mejoras, contrario a la obsolescencia, la cual se entiende como una inadecuación del sistema estudiado a las circunstancias actuales. Para realizar el diagnóstico del problema, que es la segunda etapa de este método, Easton recomienda un procedimiento de diagnóstico que consta de once pasos, el cual puede ser más simple 0 más complejo, según la naturaleza del problema. El tercer y último paso clarifica, a través de la jerarquía de objetivos, las decisiones que deben tomarse, pues se parte del supuesto de que el diagnóstico ha sido hecho y se han verificado las causas que originan la problemática en cuestión.

Para contar con una visión tanto externa como interna de los factores que inciden directamente al mal funcionamiento de un sistema, tales como estrategias, procesos operativos, productos, servicios y recursos humanos, tecnológicos y financieros, se recomienda un análisis DAFO, el cual estudia las debilidades, amenazas, fortalezas y oportunidades (Udaondo, 1998). De esta manera, los puntos fuertes internos que estén relacionados con oportunidades externas, orientan acerca de lo que debe ser impulsado por la empresa mediante estrategias bien definidas. De igual modo, el análisis comparativo de los puntos débiles y su correlación con amenazas externas, indicará en cuáles puntos se enfatizará en el momento de cambiar dicha estrategia o de introducir elementos que fortalezcan y refuercen a la empresa en sus áreas de mayor vulnerabilidad.

Para lograr un panorama más profundo de la situación problemática interna, se sugieren técnicas complementarias, como son algunos diagramas utilizados por la Administración Total de la Calidad (TQM) por sus siglas en inglés, Total Q ual ity $M$ anagement, por ejemplo: el de Ishikawa; de relaciones y de afinidad. El diagrama de Ishikawa, como es sabido, describe las relaciones entre el efecto (problema) con sus causas potenciales. El diagrama de relaciones, que tiene cierta similitud con el anterior, pertenece a la familia enunciada por Bustamante y Prudente (2001) como las "Nuevas 7 herramientas del control de calidad". Se usa para disminuir la complejidad en una situación problemática enumerando, de forma organizada y racional, las relaciones causa-efecto entre las variables que presentan algún desorden existente, y por lo tanto, serviría también para realizar un diagnóstico. Adicionalmente, se tiene el diagrama de afinidad empleado por Solís Coria (2002), cuya utilidad se concentra en la clarificación del problema en sí mismo, mediante la integración de datos obtenidos de un fenómeno complejo cualquiera.

TQM se dirige más a la administración básica que al con trol de calidad, ya que confiere un gran valor al hecho de conocer y satisfacer las necesidades de los clientes. Incluye además, cualquier aspecto del producto: procesos, diseño y sistemas de administración y control que tengan algún efecto potencial en su calidad (Hicks, 2000). 


\section{Desarrollo}

\section{Planteamiento del problema}

Se percibió una inconsistencia metodológica para diagnosticar problemas de ingeniería, a partir de tres textos que como parte fun da men tal describen la aplicación de una jerarquía de objetivos (Easton, 1978). A continuación se reseñan:

a) Artículo de revista de divulgación (Morales et al., 2001), que informa sobre la construcción de una jerarquía de objetivos en su modalidad de árbol de medios metas, para clarificar el proceso de validación de una compañía farmacéutica de la región de Orizaba. Este trabajo carece de un método de diagnóstico, o cuando menos, no se publicó.

b) El trabajo de tesis de maestría de Cruz Moreno (2002), quien resolvió la necesidad de aumentar la eficiencia de una empresa de servicios de cómputo DCASIA. Para ello, primero realizó un diagnóstico con el uso de un análisis DAFO y a partir de ahí, construyó una jerarquía de objetivos para satisfacer su problema. Como enlace diagnóstico-jerarquía, la tesista realizó un diagrama de flujo concep tual, de acuerdo a sus conocimientos y experiencia en dicha organización.

c) Otro trabajo de tesis en el que se emplea esta forma de solucionar problemas, es el realizado por Vela Cao Romero (2003), quien a partir de dos técnicas: un análisis DAFO para establecer el entorno externo e interno de la empresa Bombas Sumergibles de Córdoba, S.A. de C.V. y un diagrama causaefecto, identificó la problemática de la empresa. Como tercer y último paso, construyó la jerarquía de objetivos.

A partir del análisis de estos trabajos, se vislumbra que se puede ahondar más en la investigación de esta posible deficiencia. Por lo que se decidió detectar la familiaridad que existe por parte de los alumnos de la División de Estudios de Posgrado e Investigación del Instituto Tecnológico de Orizaba, hacia el empleo de herramientas de calidad, tales como el diagrama de Ishikawa, el diagrama de afinidad y el diagrama de relaciones que forman parte de las siete herramientas de TQM. Para ello, se realizó la aplicación de una encuesta a través de un cuestionario a cincuenta alumnos y se revisaron cincuenta trabajos de tesis de las áreas de ingeniería administrativa y de ingeniería in dus trial. A partir de ello, se obtuvieron datos que reflejaron un área de oportunidad que, desde el enfoque de este trabajo, es determinante para justificar el desarrollo del mismo: la falta de un procedimiento sistemático para realizar diagnósticos de ingeniería, no importando las herramientas que se usen posteriormente para la solución del problema identificado.

\section{Objetivo general}

Diseñar un método claro, confiable y sencillo que, tomando como base algunas técnicas de Calidad Total, disminuya la ambigüedad y la complejidad en la elaboración de un diagnóstico en ingeniería, fundamentando este proceso en la similitud de la interpretación del método científico propuesta por Van Dalen y Meyer y la metodología del inicio del proceso de decisión descrita por Easton (1978).

\section{Objetivos particulares}

- Aportar un método que ayude a capturar las necesidades de cambio de decisores potenciales.

- Aprovechar la similitud entre los pasos del método científico y el diagnóstico en ingeniería.

- Establecer una metodología para diagnosticar científicamente.

- Obtener un apoyo metodológico para hacer más ágil el inicio del proceso de decisión.

- Obtener una herramienta útil en la ingenieríaadministrativa.

- Promover el diagnóstico como parte fundamental de la aplicación de la ingeniería.

\section{Justificación}

Los resultados de este proyecto servirán para que, a partir de las percepciones que un decisor potencial tiene sobre el mal funcionamiento $u$ obsolescencia de un sistema empresarial, de una manera consistente y clara se puedan emitir 
diagnósticos relevantes; lo que permitirá encuadrar el problema adecuadamente en tiempo y forma, y así contribuir a la disminución de la complejidad en la solución de los mismos. También se piensa que el hecho de atraer la atención sobre la importancia de detectar e identificar problemas adecuadamente, influirá en los estudiantes de ingeniería de manera provechosa, evitando pérdidas de tiempo y desperdicio de recursos.

\section{Estudios y procedimientos}

Apoyándose en la visión que del méto do científico aportan Van Dalen y Meyer (1979) y la meto dología de Easton (1978), acerca del inicio del proceso de decisión, se efectuó un análisis identificando un paralelismo entre ambos procesos, hallándose las relaciones que se expresan en la figura 1.

Ambos inician con la percepción de un problema o reconocimiento de una necesidad de cambio, pero mientras que en el método científico se observa la situación problemática en forma detallada para plantear las hipótesis y posibles soluciones para verificarlas, en la ingeniería se emplean herramientas de calidad para elaborar un plan de diagnóstico. En ambos métodos existe una etapa final llamada "generación de soluciones preliminares".

\section{Metodología}

Éste se define como un proyecto de investigación cualitativa, cuyo objetivo científico principal es la comprensión de los fenómenos mediante el análisis de las percepciones e interpretaciones de los sujetos.

\section{Selección del problema Se realizó a través de la percepción del mismo}

2. Elección del diseño

Longitudinal y flexible

3. Acceso al campo

Se llevó a cábo en las instalaciones de la División de Estudios de Posgrado e Investigación del Instituto Tecnológico de Orizaba.

4. Muestra

Cincuenta tesis de las maestrías en ciencias en ingeniería administrativa e industrial desarrolladas entre los años 2000-2004 (aproximadamente el $90 \%$ de la población).

5. Selección de informantes Cincuenta estudiantes de la maestría en ciencias en ingeniería administrativa en el año 2004 (aproximadamente el $90 \%$ de la población).

6. Procedimientos para la recolección y el analisis de la información Observación directa, aplicación de encuestas y entrevistas.

Conteo.

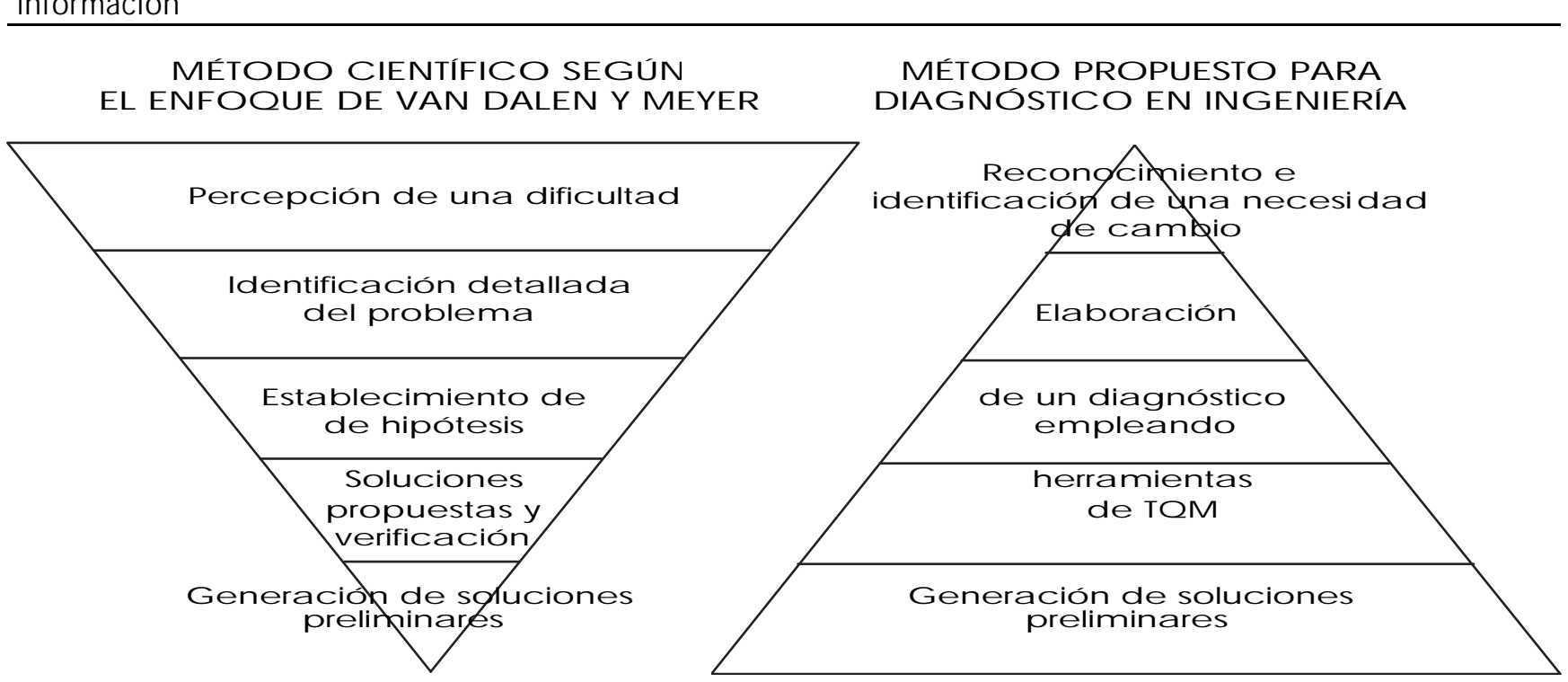

Figura 1. Diagrama comparativo entre las etapas del método científico y el diagnóstico en ingeniería propuesto 


\section{Análisis de resultados}

El método de diagnóstico aquí propuesto (Figura 2), se recomienda para dar solución a aquellos entornos empresariales que presentan áreas de oportunidad. En primer lugar y como requisito esencial para la aplicación asertiva del mismo, según experiencia de los autores de este artículo, se deben conocer la visión, misión, objetivos generales e individuales de la organización y/o del área donde se localiza la situación problemática. De no contar con ellos la empresa u organización, se recomienda enfáticamente diseñar primero estos elementos.

A continuación se explica la figura 2:

1. El método de diagnóstico empieza cuando se reconoce una necesidad de cambio, lo que equivale a percibir un problema.

2. Para que se analice correctamente el asunto a mejorar, es conveniente conocer lo mejor posible los entornos externo e interno donde éste se encuentre, por tanto se sugiere trabajar con: a) EI análisis DAFO. Con ello se analizan las potencialidades del medio en donde se originó la identificación de la necesidad del cambio, ya sea negativa o positivamente, de dos maneras diferentes: amenazas y oportunidades.

Posteriormente, el analista debe ampliar su conocimiento de todos y cada uno de los aspectos del entorno interno de la situación problemática. Esto se hará aplicando sistemáticamente las siguientes herramientas.

b) Diagrama de afinidad. Éste se usará para generar ideas sobre todas las posibles causas que originan la situación problemática y su organización lógica.

c) Diagrama causa-efecto. Será útil como apoyo para comprender las ideas y su estructura, fruto del diagrama de afinidad que refleja la relación entre el efecto (problema) con las causas potenciales que se desprenden de una problemática determinada.

\section{Reconocimiento e identific ación de la necesidad de} cambio

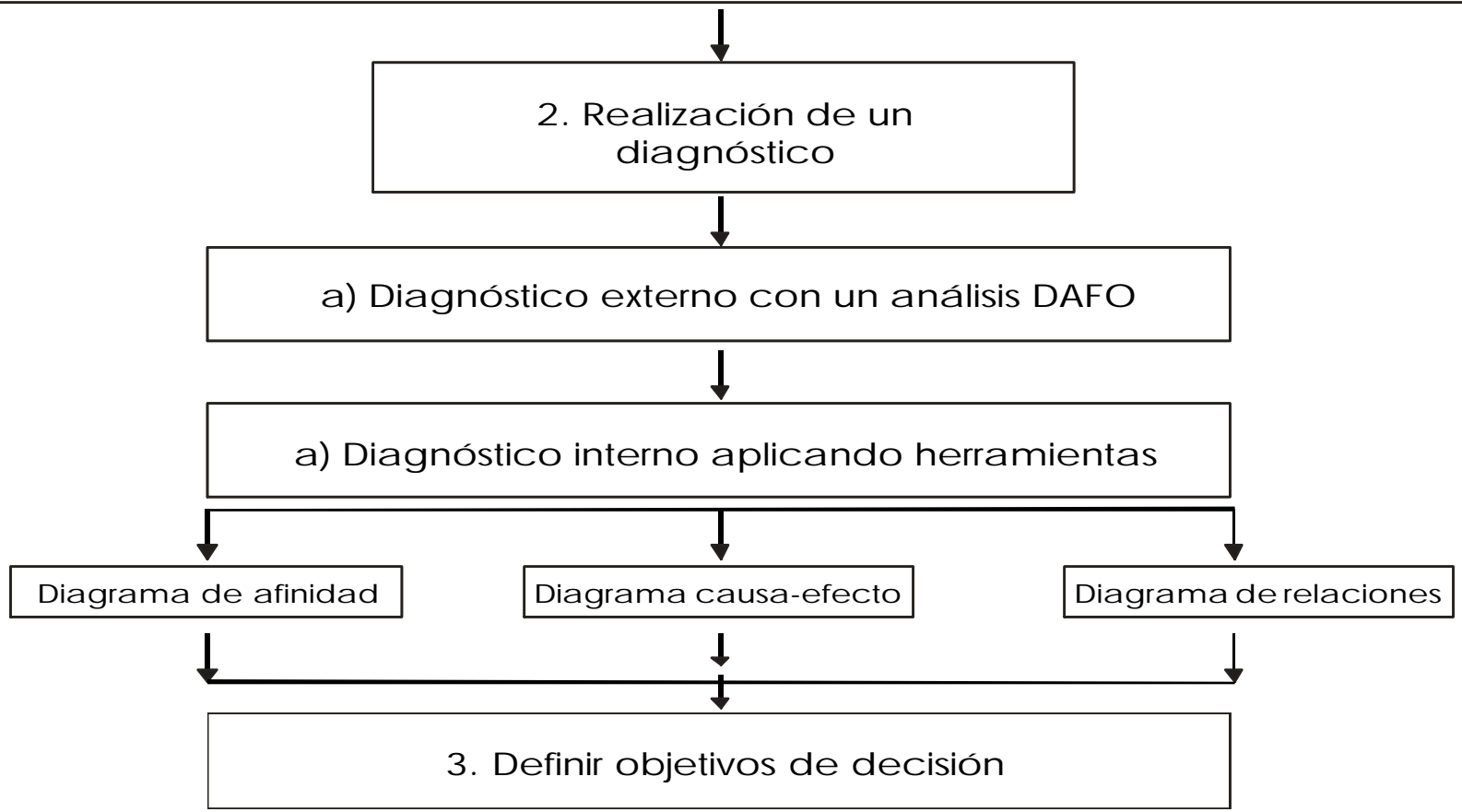

Figura 2. Proceso inte gral de diagnóstico para resolver una situación problemática (Elaboración propia, 2003) 
d) Diagrama de relaciones. Es de suma utilidad cuando se presentan problemas poco claros que cuentan con causas que se ligan entre sí de forma complicada, y como conclusión ordenada para tener como resultado un diagnóstico detallado.

El analizar las relaciones de causa-efecto por los distintos métodos, revela la importancia y el poder relativo de cada una de ellas, así como su interrelación dinámica.

Al concluir con la aplicación de las herramientas, se deben haber obtenido la generación de soluciones preliminares.

\section{Conclusiones y recomendaciones}

Mediante este método se ofrece una técnica a los decisores potenciales de las empresas, la cual les ayuda a capturar sus percepciones sobre necesidades de cambio y aprovechar éstas para promover el diagnóstico como parte fundamental de la aplicación de la ingeniería.

La similitud entre los pasos del método científico y el diagnóstico en ingeniería, permite aprovechar los recursos de una manera más racional, que va de acuerdo con el pensamiento científico. Esto servirá como apoyo metodológico para hacer más ágil el inicio del proceso de decisión. Esperamos que esta herramienta sea cada vez más usada en la ingeniería administrativa, evitando pérdidas de tiempo y soluciones equivocadas en el ámbito empresarial.

\section{Referencias}

Aiteco (2003). "Diagrama causa-efecto", obtenido de la Red Mundial el 7 de Febrero, http://www.aiteco.com/ishikawa.htm.

Bustamante A. y Prudente E. (2001). Las nuevas siete herramientas del control de calidad. Revista UPIICSA, mayo-agosto, pp. 31-35.

Cucei (2003). "Diagrama de ishikawa", métodos para su construcción, obtenido de la Red M undial el 8 de febrero,

http://www.cucei.udg.mx/ luisdegu/calida d_total/Unidad_2/herbas/ishi1/ishil.html.

Cruz-Moreno A.M. (2001). Modelo de control interno para incrementar la eficiencia en centros de computo de la iniciativa privada. Tesis de Maestría en Ciencias en Ingeniería Administrativa, Instituto Tecnológico de Orizaba, México.

Easton A. (1978). Decisiones administrativas con objetivos múltiples. Editorial Limusa, México, DF.

Hicks P.E. (2000). Ingeniería Indus trial y Administración. Edito rial CECSA, México, DF.

Ishikawa K. (1993). ¿Qué es control total de calidad? Edito rial No rma, Colombia.

(2003). "Las 7 herramientas de control de calidad", obtenido de la Red Mundial el 10 de febrero, http://www.geo cities.com/WallStreet/excha nge/9158/7herra.htm.

(2003). "Las nuevas 7 herramientas de la calidad", obtenido de la Red Mundial el 10 de febrero http://www.geocities. com/CapeCanaveral/Hangar/4434//7nh.html.

(2003). "Las nuevas 7 herramientas de la calidad", obtenido de la Red Mundial el 10 de febrero, http://www. woerldclassconsultant.org/serv0 3.htm

Morales G., Quiroz F. y Ramírez-Carrera L.C. (2001). Aplicación de un árbol de medios y metas al proceso de validación de una compañía farmacéutica. Revista UPIICSA, mayo-agosto, pp. 19-25.

Solís-Coria M.L. (2002). Propuesta de un modelo de investigación para el Colegio de Bachilleres. Tesis de Maestría en Ciencias en Ingeniería Administrativa, Instituto Tecnológico de Orizaba, México.

Udaondo M. (1998). G estión de la calidad. Editorial Díaz de Santos, S.A., España.

Van-Dalen D. y Meyer W.J. (1979). Manual de técnica de la investigación educacional. Edito rial Limusa, México.

Vela-Cao-Romero L. (2002). Diseño y aplicación de una Jerarquía de objetivos para la empresa Bombas Sumergibles de Córdoba S.A. de C. V. Tesis de Licenciatura en Ingeniería Industrial, Instituto Tecnológico de Orizaba, México. 
DOI: http://dx.doi.org/10.22201/fi.25940732e.2005.06n2.010

Del método científico al diagnóstico de problemas de ingeniería en México

\section{Semblanza de los autores}

O scar Ernesto Sotelo-Rosas. Es ingeniero indus trial por el Instituto Tecnológico de Orizaba, logró el título profesional por alto promedio, obtuvo el grado de maestro en ciencias en ingeniería administrativa por el Instituto Tecnológico de Orizaba, alcanzó este nivel por su trabajo de tesis llamado "Diseño, construcción y aplicación de un método que gestione la solución de problemas de decisión", del cual se desprende esencialmente este artículo. También es icenciado en informática y técnico en construcción egresado del Centro de Bachillerato Tecnológico indus trial y de servicios 142.

Lilia C. Ramírez-Carrera. Es licenciada en ingeniería química por la Facultad de Ciencias Químicas de la Universidad Veracruzana, obtuvo la maestría en ciencias en ingeniería indus trial por el Instituto Tecnológico de Orizaba. Asimismo, es especialista en docencia por el Centro Interdisciplinario de Investigación y Docencia en Educación Técnica de Querétaro, Qro. Actualmente se desempeña como profesora del posgrado en el Instituto Tecnológico de Orizaba. 